\title{
THEORETICAL CONSIDERATION OF CHARGE SIMULATION METHOD FOR NUMERICAL CONFORMAL MAPPINGS IN A RING DOMAIN
}

\author{
TETSUO INOUE \\ Department of Applied Mathematics, Kobe Mercantile Marine College \\ Higashinada, Fukae-Minami 5-1-1, Kobe 658, Japan
}

(Received August 16, 1996 and in revised form March 17, 1997)

\begin{abstract}
The charge simulation method has been applied to solve a lot of problems in electrical engineering. However, the principle of the method is not known enough even now. This paper is devoted to giving the theoretical and mathematical base for the charge simulation method of numerical conformal mappings in ring domains. Therefore for example, the uniform .convergence of approximations, the theoretical distribution of charge points, and the charges will be mathematically discussed. An example is shown to help understanding of theoretical considerations.
\end{abstract}

KEYWORDS AND PHRASES. Charge Simulation method, Weighted asymptotic theorem, Potential theory, Hyperbolic polynomial, Conformal mapping, Uniform convergence

1991 AMS SUBJECT CLASSIFICATION CODE. 30E10, 41A10, 65 E05.

\section{INTRODUCTION}

The charge simulation method is very useful to solve partial differential equations in electrical engineering, and has been studied and developed by a lot of researchers. The method is easy to understand, and can be applied only by solving a system of simultaneous linear equations. Many examples show that the method makes possible to get rather precise solutions for the boundary value problems with respect to domains bounded by smooth curves.

However, many parts of the method depend on the experiment from the examles. For instance. the best distribution of charge points is not known.

In this paper we will give the theoretical and mathematical base for the charge simulation method of numerical conformal mappings in ring domains.

Then, the potential theory $[10,16]$, especially the asymptotic theorems $[5-9,11-14]$ on extremal weighted hyperbolic polynomials play fundamental roles. The theorems depend on the notion introduced in 1992 by Mhaskar and Saff [13]. We note that numerical conformal mappings not depending on the charge simulation method have been shown in $[4,15]$.

The results established in this paper are as follows:

(1a) A new scheme for approximations is proposed for the numerical conformal mapping of a ring domain.

(1b) The ditribution of charge points is characterized by the weighted extremal points.

(1c) It is theoretically shown that the numbers of charge points in the complements of a ring domain must be equal to each another. 
(1d) If the outer boundary is the unit circle with a center at the origin, the charge points exterior to it are represented by $\left\{1 / \bar{z}_{n, 1}\right\}_{i=1}^{n}$, where $\left\{z_{n, 1}\right\}_{i=1}^{n}$ are the charge points interior to the inner boundary.

(1e) The approximations converge uniformly in the domain and on the boundary, if the charge points are theoretically distributed.

The outline of this paper is as follows. In sections 2 and 3, the definition of weighted hyperbolic capacity (shortly $w h$-capacity) and asymptotic lemmas on extremal $w h$-polynomials will be introduced, respectively. In section 4 the lemmas are applied to establishing algorithms of a new charge simulation method for numerical conformal mappings in ring domains whose each outer boundary is the unit circle with a center at the origin. In section 5 the theoretical distribution of charge poins is discussed. In section 6 a simple example is shown to help understanding of theoretical considerations for the new charge simulatin method.

\section{DEFINITIONS}

In approximation theory, the asymptotic behavior of extremal polynomials has been studied by a lot of researchers. In this section we describe the notions of weighted hyperbolic polynomials (shortly $w h$-polynomials) and weighted hyperbolic capacity (wh-capacity) introduced by the author [5] and Mhaskar-Saff [12,13], respectively.

Let $D$ be a ring domain whose outer and inner boundaries are the unit circle $\gamma_{0}$ with a center at the origin and a Jordan curve $\gamma$, respectively. For the numerical conformal mapping of a ring domain we establish a new charge simulation method depending on asymptotic lemmas on extremal weighted hyperbolic polynomials. There the distance between $z_{\imath}$ and $z$, is defined by

$$
\left|\frac{z_{\imath}-z_{\jmath}}{1-\bar{z}_{\jmath} z_{\imath}}\right| w\left(z_{\imath}\right) w\left(z_{\jmath}\right)
$$

where $w(z)$ is a weight function.

All of the definitions for the usual weighted polynomials hold analogously for hyperbolic ones. Let $w=w(z)$ be an arbitrary, continuous, positive function defined on the complex plane $C$. For each integer $n \geq 1$, we let $P_{n, w}$ denote the class of all polynomials of the form

$$
p_{n, w}(z)=\prod_{i=1}^{n}\left[\left(\frac{z-z_{n, 1}}{1-\bar{z}_{n, 2} z}\right) w(z) w\left(z_{n, 2}\right)\right],
$$

which we call $w h$-polynomials of degree $n$.

Let $M(\gamma)$ denote the class of all positive unit Borel measures whose support is $\gamma$. We define wh-capacity by

$$
\operatorname{caph}(w, \gamma)=\exp \left(V_{w}\right)
$$

where

$$
I_{w}(\sigma)=\iint \log \left[\left|\frac{z-t}{1-\bar{t} z}\right| w(z) w(t)\right] d \sigma(z) d \sigma(t)
$$

and

$$
V_{w}=V(w, \gamma)=\sup _{\sigma \in M(\gamma)} I_{w}(\sigma) .
$$

Throughout the remainder of this paper, we assume that every $p_{n, w}(z)$ has all of the zeros on $\gamma$.

Though this assumption is rather strong, it is the key point to establish the new charge simulation method. 
Let $\mu_{w} \in M(\gamma)$ be an extremal measure such that

$$
I_{w}\left(\mu_{w}\right)=V_{w} .
$$

The existence and the uniqueness of $\mu_{w}$ were shown in [13,Theorem 3.1(b)]. We assume that $S_{w}=\gamma$, where $S_{w}$ is the support of $\mu_{w}$.

For polynomials $p_{n, w}(z)$ of degree $n$, the discrete unit measure defined on compact sets in the complex plane $C$ with mass $1 / n$ at each zero of $p_{n, w}(z)$ will be denoted by $\mu_{n, w}=\mu\left(p_{n, w}\right)$. It will be called the normalized counting measure on the zeros of $p_{n, w}(z)$. If $p_{n, w}(z)$ has multiple zeros, the obvious modification will be considered.

The weak convergence of $\nu_{n}$ to $\nu$ as $n \rightarrow \infty$ will be defined by

$$
\lim _{n \rightarrow \infty} \int f d \nu_{n}=\int f d \nu
$$

for every continuous function in the complex plane $C$ with compact support.

\section{LEMMAS}

We present the fundamental lemmas on extrmal $w h$-polynomials that are devoted to mathematical considerations of the new charge simulation method.

Since all of the lemmas established for usual weighted polynomials hold analogously for hyperbolic ones, we omit the proofs (see [5-9,11-14]).

Under the assumption mentioned in section 2, we state the main lemma which has been verified in [6].

Lemma 3.1. Let

$$
p_{n, w}(z)=\prod_{i=1}^{n}\left[\left(\frac{z-z_{n, i}}{1-\bar{z}_{n, \imath} z}\right) w(z) w\left(z_{n, \imath}\right)\right]
$$

be wh-polynomials of degree $n$ satisfying the conditions $(3 a)$ and $(3 b)$ below:

(3a) $\lim \sup _{n \rightarrow \infty}\left\|p_{n, w}(z)\right\|_{\gamma}^{1 / n} \leq \operatorname{caph}(w, \gamma)$.

(3b) $\liminf _{n \rightarrow \infty} \frac{1}{n} \sum_{s=1}^{n} \log w\left(z_{n, 2}\right) \geq \int \log w(t) d \mu_{w}(t)$.

Then, there holds the equality

$$
\lim _{n \rightarrow \infty}\left|p_{n, w}(z)\right|^{1 / n}=\exp \left\{g_{w}(z)\right\}
$$

uniformly on every compact subset of $D$, where

$$
g_{w}(z)=\int \log \left[\left|\frac{z-t}{1-\bar{t} z}\right| w(z) w(t)\right] d \mu_{w}(t) .
$$

We note that if (3.1) holds uniformly on every compact subset of $D$, the property $(3 b)$ follows without inf and with equality. It is easily shown by letting $z$ tend to 1 in (3.1).

Now, we show the converse of Lemma 3.1 .

Lemma 3.2. Let

$$
p_{n, w}(z)=\prod_{i=1}^{n}\left[\left(\frac{z-z_{n, i}}{1-\bar{z}_{n, 2} z}\right) w(z) w\left(z_{n, 2}\right)\right]
$$

be wh-polynomials of degree $n$ satisfying the equality

uniformly on every compact subset of $D$.

$$
\lim _{n \rightarrow \infty}\left|p_{n, w}(z)\right|^{1 / n}=\exp \left\{g_{w}(z)\right\}
$$

Then, there hold the relations

(3a) limsup $\sup _{n \rightarrow \infty}\left\|p_{n, w}(z)\right\|_{\gamma}^{1 / n} \leq \operatorname{caph}(w, \gamma)$.

$\left(3 b^{\prime}\right) \lim _{n \rightarrow \infty} \frac{1}{n} \sum_{i=1}^{n} \log w\left(z_{n, 2}\right)=\int \log w(t) d \mu_{w}(t)$.

With the above notation we state a well-known theorem on the zeros distribution of extremal polynomials which has been established in [12,p.88-89]. 
Lemma 3.3. Let

$$
p_{n, w}(z)=\prod_{i=1}^{n}\left[\left(\frac{z-z_{n, i}}{1-\bar{z}_{n, 2}}\right) w(z) w\left(z_{n, 2}\right)\right]
$$

and $\mu_{n, w}=\mu\left(p_{n, w}\right)$ be wh-polynomials of degree $n$ and the normalized counting measure of $p_{n, w}$ respectively, satisfying the equality

$$
\lim _{n \rightarrow \infty}\left|p_{n, w}(z)\right|^{1 / n}=\exp \left\{g_{w}(z)\right\}
$$

uniformly on every compact subset of $D$. Then, there holds

$(A) ; \mu_{n, w}$ converges weakly to $\mu_{w}$ as $n \rightarrow \infty$.

In fact, Lemma 3.3 has been proved for the case when $\gamma$ lies on the real axis and for the $w$-polynomials

$$
s_{n, w}(z)=\prod_{\imath=1}^{n}\left[\left(\frac{z-z_{n, i}}{1-\bar{z}_{n, 2} z}\right) w(z)\right] .
$$

Since the proof for the case of Lemma 3.3 is similar, we will omit it.

The following theorem has recently established by the author.

Lemma 3.4. Let $\mu_{n, w}$ converge weakly to $\mu_{w}$ as $n \rightarrow \infty$. Then, we have

$$
\limsup _{n \rightarrow \infty}\left\|p_{n, w}(z)\right\|_{\gamma}^{1 / n} \leq \operatorname{caph}(w, \gamma) .
$$

From Lemmas 3.3 and 3.4 we have the following lemma under the assumption mentioned above.

\section{Lemma 3.5.}

$$
\lim _{n \rightarrow \infty}\left|p_{n, w}(z)\right|^{1 / n}=\exp \left\{g_{w}(z)\right\}
$$

holds uniformly on every compact subset of $D$ if and only if $(A)$ holds.

We transform (3.3) to the form

$$
\lim _{n \rightarrow \infty}\left|\prod_{\imath=1}^{n}\left(\frac{z-z_{n, 2}}{1-\bar{z}_{n, 2} z}\right) w\left(z_{n, 2}\right)\right|^{1 / n}=\exp \left\{\int \log \left[\left|\frac{z-t}{1-\bar{t} z}\right| w(t)\right] d \mu_{w}(t)\right\} .
$$

It is known that the equality

$$
\exp \left\{\int \log \left[\left|\frac{z-t}{1-\bar{t} z}\right| w(z) w(t)\right] d \mu_{w}(t)\right\}=\operatorname{caph}(w, \gamma)
$$

holds quasi-everywhere (q.e.) on $\gamma$ [13]; that is, with the possible exception of a set having capacity zero. Therefore, we obtain the equalities

$$
\begin{aligned}
& \frac{\exp \left\{\int \log [|(z-t) /(1-\bar{t} z)| w(t)] d \mu_{w}(t)\right\}}{\operatorname{caph}(w, \gamma)} \\
= & \frac{\exp \left\{\int \log [|(z-t) /(1-\bar{t} z)| w(z) w(t)] d \mu_{w}(t)\right\}}{w(z) \operatorname{caph}(w, \gamma)} \\
= & \frac{1}{w(z)}
\end{aligned}
$$

q.e. on $\gamma$. Combining (3.10) and Lemma 3.5, we have the following 


\section{Lemma 3.6.}

$$
\lim _{n \rightarrow \infty}\left\{\prod_{i=1}^{n}\left|\left(\frac{z-z_{n, 2}}{1-\bar{z}_{n, 2} z}\right)\right| w\left(z_{n, \imath}\right)\right\}^{1 / n}=\exp \left\{\int \log \left[\left|\frac{z-t}{1-\bar{t} z}\right| w(t)\right] d \mu_{w}(t)\right\}
$$

holds uniformly on every compact subset of $D$ if and only if $(A)$ holds. Furthermore, if it is satisfied, the equality

$$
\frac{\exp \left\{\int \log [|(z-t) /(1-\bar{t} z)| w(t)] d \mu_{w}(t)\right\}}{\operatorname{caph}(w, \gamma)}=\frac{1}{w(z)}
$$

holds q.e. on $\gamma$.

Let $D^{\prime}$ be a ring domain whose outer and inner boundaries are the unit circle $\gamma_{0}^{\prime}$ with a center at the origin and a Jordan curve $\gamma^{\prime}$, respectively.

We assume that the function $w=f(z)$ maps $D \cup \gamma_{0}$ onto $D^{\prime} \cup \gamma_{0}^{\prime}$, corresponding $\gamma$ to $\gamma^{\prime}$. The function $f(z)$ is uniquely determined under the condition $f(1)=1$. When the domain $D$ is given, the modulus of $D$ is uniquely determined [1].

The asymptotic formula for the $n$th root of $p_{n, w}(z)$ can be obtained from one of $\left|p_{n, w}(z)\right|$. This follows from the fact that

$$
\log \left(p_{n, w}(z)\right)^{1 / n}=\log \left|p_{n, w}(z)\right|^{1 / n}+i \arg \left(p_{n, w}(z)\right)^{1 / n}
$$

is analytic in $D$ and Cauchy-Riemann relation holds between the real and imaginal parts [14].

Thus, using Lemma 3.6 with $w(z)=1 /|f(z)|$ the asymptotic formula for $f(z)$ can be obtained from the $n$th root of the weighted polynomial

$$
\prod_{i=1}^{n}\left[\left(\frac{z-z_{n, 2}}{1-\bar{z}_{n, 2} z}\right) w\left(z_{n, 2}\right)\right]
$$

and $\operatorname{caph}(w, \gamma)$.

Lemma 3.7. Under the condition $(A)$, the approximation $f_{n}(z)$ of $f(z)$ can be obtained by

$$
f_{n}(z)=e^{i \theta_{n}}\left\{\prod_{i=1}^{n} \frac{z-z_{n, 2}}{1-\bar{z}_{n, 2} z}\right\}^{1 / n}\left(\theta_{n} ; \text { real }\right)
$$

for a sufficiently large number $n$. Then $f_{n}(z)$ converges to $f(z)$ uniformly on every compact subset of $D . \theta_{n}$ is determined by the condition $f_{n}(1)=1$.

\section{ALGORITHMS}

Using the terminologies of the charge simulation method, Lemma 3.7 is mentioned as follows for the numerical conformal mapping of a ring domain.

Algorithm 4.1. The approximation $f_{n}(z)$ of $f(z)$ is obtained as follows:

(4a) The charge points $\left\{z_{n, 2}\right\}_{\imath=1}^{n}$ satisfying the condition $(A)$ are chosen on $\gamma$.

(4b) The charge at every charge point is assumed to be $1 / n$.

(4c) The approximation $f_{n}(z)$ of $f(z)$ can be obtained by

$$
f_{n}(z)=e^{i \theta_{n}}\left\{\prod_{i=1}^{n} \frac{z-z_{n, 2}}{1-\bar{z}_{n, 2} z}\right\}^{1 / n}\left(\theta_{n} ; \text { real }\right)
$$

for a sufficiently large number $n$. Then $f_{n}(z)$ converges to $f(z)$ uniformly on every compact subset of $D$. $\theta_{n}$ is determined by the condition $f_{n}(1)=1$.

Algorithm 4.1 suggests us the following algorithm for the charge simulation method on the numerical conformal mapping of a ring domain. 
Algorithm 4.2. The approximation $f_{n}(z)$ of $f(z)$ is obtained as follows:

(4d) The charge points $\left\{z_{n, i}\right\}_{i=1}^{n}$ are 'theoretically' chosen interior to $\gamma$.

(4e) The charge at every charge point is assumed to be $1 / n$.

(4f) The approximation $f_{n}(z)$ is represented by

$$
f_{n}(z)=e^{i \theta_{n}}\left\{\prod_{i=1}^{n} \frac{z-z_{n, i}}{1-\bar{z}_{n, 2} z}\right\}^{1 / n}\left(\theta_{n} ; \text { real }\right)
$$

for a sufficiently large number $n$. Then $f_{n}(z)$ converges to $f(z)$ uniformly on every compact subset of $D \cup \gamma . \theta_{n}$ is determined by the condition $f_{n}(1)=1$.

Algorithm 4.2 suggests us the following

Algorithm 4.3. The approximation $f_{n}(z)$ of $f(z)$ is obtained as follows:

(4g) The charge points $\left\{z_{n, i}\right\}_{i=1}^{n}$ and the collocation points $\left\{\zeta_{n, i}\right\}_{i=1}^{n}$ are 'appropriately' chosen inteior to $\gamma$ and on $\gamma$, respectively.

(4h) When $\alpha_{1}(i=1,2, \ldots, n)$ are the solutions of a system of simultaneous linear equations

$$
\begin{aligned}
& \sum_{i=1}^{n} \alpha_{\imath} \log \left|\frac{\zeta_{n, k}-z_{n, 2}}{1-\bar{z}_{n, 2} \zeta_{n, k}}\right|=\log \left|f\left(\zeta_{n, k}\right)\right| \quad(k=1,2, \ldots, n), \\
& \alpha_{1}+\alpha_{2}+\cdots+\alpha_{n}=1
\end{aligned}
$$

the charges at $\left\{z_{n, 2}\right\}_{i=1}^{n}$ are given by $\left\{\alpha_{\imath}\right\}_{i=1}^{n}$.

(4i) The approximation $f_{n}(z)$ is represented by

$$
f_{n}(z)=e^{i \theta_{n}} \prod_{i=1}^{n}\left(\frac{z-z_{n, 2}}{1-\bar{z}_{n, z} z}\right)^{\alpha_{i}} \quad\left(\theta_{n} ; \text { real }\right)
$$

for a sufficiently large number $n$. $\theta_{n}$ is determined by the condition $f_{n}(1)=1$.

Algorithm 4.3 requires $n-1$ values $\left|f\left(\zeta_{n, k}\right)\right|$ within $k=1,2, \ldots, n$ to be given. However, all of the values are not required when the image domain $D^{\prime}$ is a disk with a center at the origin (only the value $\left|f\left(\zeta_{n, k}\right)\right|=\rho(k=1,2, \ldots, n)$ is not known). From Algorithm 4.3 for this case, we have the following

Algorithm 4.4. The approximation $f_{n}(z)$ of $f(z)$ is obtained as follows:

(4j) The charge points $\left\{z_{n, t}\right\}_{i=1}^{n}$ and the collocation points $\left\{\zeta_{n, 2}\right\}_{i=1}^{n}$ are appropriately chosen inteior to $\gamma$ and on $\gamma$, respectively.

(4k) When $\alpha_{i}(i=0,1,2, \ldots, n)$ are the solutions of a system of simultaneous linear equations

$$
\begin{aligned}
& \sum_{i=1}^{n} \alpha_{i} \log \left|\frac{\zeta_{n, k}-z_{n, 2}}{1-\bar{z}_{n, 2} \zeta_{n, k}}\right|=\alpha_{0} \quad(k=1,2, \ldots, n), \\
& \alpha_{1}+\alpha_{2}+\cdots+\alpha_{n}=1
\end{aligned}
$$

the charges at $\left\{z_{n, 1}\right\}_{i=1}^{n}$ are given by $\left\{\alpha_{i}\right\}_{i=1}^{n}$.

(4l) The approximation $f_{n}(z)$ is represented by

$$
f_{n}(z)=e^{i \theta_{n}} \prod_{i=1}^{n}\left(\frac{z-z_{n, 2}}{1-\bar{z}_{n, 2} z}\right)^{\alpha_{i}} \quad\left(\theta_{n} ; \text { real }\right)
$$

for a sufficiently large number $n$. $\theta_{n}$ is determined by the condition $f_{n}(1)=1$.

The numerical experiments show that the approximations

$$
\alpha_{0} \simeq \log \rho, \quad \alpha_{i} \simeq \frac{1}{n}(i=1,2, \ldots, n)
$$


with

$$
\rho=\operatorname{caph}\left(1, \gamma_{\rho}\right)
$$

hold, when the charge points and the collocation points are theoretically distributed. The method introduced in this section has the following advantages compared to the standard one:

(4m) The charge points

$$
\left\{\frac{1}{\bar{z}_{n, 2}}\right\}_{i=1}^{n}
$$

exterior to $\gamma_{0}$ is uniquely determined by ones $\left\{z_{n, 1}\right\}_{i=1}^{n}$ interior to $\gamma_{\rho}$.

(4n) The number of charge points exterior to $\gamma_{0}$ is equal to one of them interior to $\gamma_{\rho}$.

(4o) When the charge points on the analytic curve whose image is a circle with center at the origin, typical examples show that numerical results of high accuracy can be obtain.

\section{DISTRIBUTION OF CHARGE POINTS}

In this section we show two kinds of weighted extremal points satisfying the condition $(A)$.

For each integer $n \geq 1$, let $Q_{n, w}$ be a set of $w h$-polynomials

$$
q_{n, w}(z)=\prod_{\imath=1}^{n}\left[\left(\frac{z-z_{n, 2}}{1-\bar{z}_{n, 2} z}\right) w(z) w\left(z_{n, 2}\right)\right],
$$

where the zeros $\left\{z_{n, 1}\right\}_{i=1}^{n}$ lie on the boundary $\gamma$ of $D$.

Let $q_{n, w}^{*}(z)$ be a polynomial such that

$$
\left\|q_{n, w}^{*}(z)\right\|_{\gamma}=\inf _{q_{n, w} \in Q_{n, w}}\left\|q_{n, w}(z)\right\|_{\gamma} .
$$

The existence of $q_{n, w}^{*}(z)$ is easily proved by the usual method. Then, $q_{n, w}^{*}(z)$ is called a $w h$ Chebyshev polynomial with zeros on $\gamma$. It is known that it satisfies the condition $(A)[5]$.

To show another polynomial satisfying the condition $(A)$, we introduce the definition of $w h$ transfinite diameter. For each integer $n \geq 2$, let

$$
\begin{aligned}
\delta_{n}(w, \gamma) & =\sup _{z_{n, 1}, \quad z_{n, n} \in \gamma}\left\{\prod_{1 \leq i<j \leq n}\left[\left|\frac{z_{n, 1}-z_{n, J}}{1-\bar{z}_{n, j} z_{n, 1}}\right| w\left(z_{n, i}\right) w\left(z_{n, j}\right)\right]\right\}^{2 /(n(n-1))} \\
& =\left\{\prod_{1 \leq i<j \leq n}\left[\left|\frac{z_{n, 2}^{*}-z_{n, j}^{*}}{1-\bar{z}_{n, j}^{*}, z_{n, 2}^{*}}\right| w\left(z_{n, 2}^{*}\right) w\left(z_{n, j}^{*}\right)\right]\right\}^{2 /(n(n-1))} .
\end{aligned}
$$

The sequence $\left\{\delta_{n}(w, \gamma)\right\}_{n=1}^{\infty}$ converges and

$$
\tau(w, \gamma)=\lim _{n \rightarrow \infty} \delta_{n}(w, \gamma)
$$

is called $w h$-transfinite diameter of $\gamma$. It is said that $\left\{z_{n, 2}^{*}\right\}_{t=1}^{n}$ are $w h$-Fekete points. It is then known that $w h$-polynomials

$$
k_{n, w}(z)=\prod_{j=1}^{n}\left[\left(\frac{z-z_{n, j}^{*}}{1-\bar{z}_{n, j}^{*} z}\right) w(z) w\left(z_{n, j}^{*}\right)\right]
$$

satisfy the condition $(A)[11]$.

\section{NUMERICAL EXPERIMENTS}

In this section, a simple example is shown to help understanding of theoretical considerations for the new method introduced in the previous section. 
Let

$$
f(z)=\frac{z-a}{1-a z} \quad(0<a<1) .
$$

This function maps conformally a domain $D$ onto

$$
\{w ; \rho<|w|<1\}
$$

where $D$ is a ring domain whose outer boundary is the unit circle.

Let $f(z)$ map a ring domain $D_{\rho}$ bounded by $\gamma_{0}$ and a Jordan curve $\gamma_{\rho}$ onto an annulus $\{w ; 1>|w|>\rho\}$ bounded by $\gamma_{0}$ and a Jordan curve $\gamma_{\rho}^{\prime}$, corresponding $\gamma_{\rho}$ to $\gamma_{\rho}^{\prime}$ with $\rho>0$.

The inverse function of $f(z)$ is

$$
z=h(w)=\frac{w+a}{1+a w} .
$$

The charge points $\left\{z_{n, 1}\right\}_{i=1}^{n}$ on $\gamma_{\rho}$ and the collocation points $\left\{\zeta_{n, 2}\right\}_{t=1}^{n}$ on $\gamma$ are chosen as the images of the points

$$
\rho \exp \left(\frac{2 \pi j(i-1)}{n}\right), j=\sqrt{-1}
$$

being distributed on $|w|=\rho$ under the mapping $z=h(w)$. We consider the case where $\rho=0.1$ and 0.6. It is known that these points are uniformly distributed (which means that these points satisfy the condition $(A))$ on the circles $\gamma_{\rho}$ and $\gamma$, respectively [3].

For $n=10$, the charge points with $\rho=0.1$ and the collocation points with $\rho=0.6$, we solve a system of simultaneous linear equations (4.6) and (4.7) and obtain the following results (to know the distribution precisely, all of the charges are denoted):

Table 1: Charges by Algorithm 4.4 for $n=10$

\begin{tabular}{|c|c|c|c|c|c|}
\hline$\alpha_{0}$ & $-0.51082563 \mathrm{E}+00$ & $\alpha_{1}$ & $0.10000704 \mathrm{E}+00$ & $\alpha_{2}$ & $0.10000123 \mathrm{E}+00$ \\
\hline$\alpha_{3}$ & $0.99986970 \mathrm{E}-01$ & $\alpha_{4}$ & $0.10002397 \mathrm{E}+00$ & $\alpha_{5}$ & $0.99968159 \mathrm{E}-01$ \\
\hline$\alpha_{6}$ & $0.10003428 \mathrm{E}+00$ & $\alpha_{7}$ & $0.99970734 \mathrm{E}-01$ & $\alpha_{8}$ & $0.10001742 \mathrm{E}+00$ \\
\hline$\alpha_{9}$ & $0.99997138 \mathrm{E}-01$ & $\alpha_{10}$ & $0.99993097 \mathrm{E}-01$ & ${ }^{*}$ & $* * * * *$ \\
\hline
\end{tabular}

The solution satisfies the relation (4.9)

$$
\alpha_{0} \simeq \log 0.6=-0.510825623 \ldots, \quad \alpha_{\imath} \simeq \frac{1}{10}(i=1,2, \ldots, 10)
$$

with high accuracy (it is remarkable that the number of charge points is only 10).

Using the charges $\left\{\alpha_{\imath}\right\}_{i=0}^{n}$ obtained above, the approximations (4.8) are represented. The accuracy of the errors are estimated by the maximum of

$$
\left|f_{n}\left(\zeta_{n, i+1 / 2}\right)-f\left(\zeta_{n, 2+1 / 2}\right)\right|
$$

where $\zeta_{n, 2+1 / 2}$ is the middle point between $\zeta_{n, 2}$ and $\zeta_{n, 1+1}$. In fact, we must estimate the errors also at the collocation points. However, we can obtain anlogous results there.

Applying reflexion principle and maximum principle, the error on the unit circle will be estimated by one on the inner boundary $\gamma_{\rho}$. Then, the accuracy may decrease by order of $10^{-1}$ at most.

We obtain the errors as follows (to know the distribution precisely, all of the errors are denoted): 
Table 2: Errors of (4.8) for $n=10$

\begin{tabular}{|c|c|c|c|}
\hline $4.4703483 \mathrm{E}-08$ & $6.6640019 \mathrm{E}-08$ & $1.2435162 \mathrm{E}-07$ & $1.1920929 \mathrm{E}-07$ \\
\hline $1.1920929 \mathrm{E}-07$ & $8.4293693 \mathrm{E}-08$ & $1.1920929 \mathrm{E}-07$ & $9.2534291 \mathrm{E}-08$ \\
\hline $8.4293693 \mathrm{E}-08$ & $5.9604644 \mathrm{E}-08$ & $* * * * * *$ & $* * * * *$ \\
\hline
\end{tabular}

Applying Algorithm 4.2 with $n=10$, using the above distribution of charge points and the theoretical values

$$
\alpha_{0}=\log 0.6, \alpha_{i}=\frac{1}{10}(i=1,2, \ldots, 10),
$$

the errors $\left|f_{n}(z)-f(z)\right|$ of the approximation (4.2) are estimated. Then the errors are as follows:

Table 3: Errors of (4.2) for $n=10$

\begin{tabular}{|c|c|c|c|}
\hline $1.2731556 \mathrm{E}-07$ & $1.0745380 \mathrm{E}-07$ & $8.2987058 \mathrm{E}-08$ & $1.2287812 \mathrm{E}-07$ \\
\hline $7.4505805 \mathrm{E}-08$ & $1.2731556 \mathrm{E}-07$ & $1.4901161 \mathrm{E}-07$ & $1.4790153 \mathrm{E}-07$ \\
\hline $1.9992004 \mathrm{E}-07$ & $6.6640019 \mathrm{E}-08$ & $* * * * *$ & $* * * * *$ \\
\hline
\end{tabular}

For the above example we have used the parameter $\rho=0.1$ for the charge points. If the charge points are not too near the boundary, analogous accuracy is obtained.

The numerical caluculation has been performed by Runfort-f77 (PC98-486AV) and single precision.

\section{REMARKS}

We have shown that the method introduced in this papar has the advantages copared to the standard one, when the charge points are theoretically distributed.

Amano's method [2] for the conformal mapping from a general ring domain to a standard one is applicable, even when the theoretical distribution is not known. It has also been shown that the approximations with high accuracy can be obtained.

Algorithm 4.4 is applicable when the charge points and collocation points are distributed by the way of Amano.

We wish that this paper would contribute to presenting the theoretical base also for Amano's method.

\section{REFERENCES}

[1] AHLFORS L.V., Complex Analysis, 2nd. ed., McGraw-Hill, New York, 1966.

[2] AMANO K., A charge simulation method for the numerical conformal mapping of interior, exterior and doubly-connected domains, J. Comput. App. Math., 53 (1994), 353-370.

[3] GAIER D., Lecture on Complex Approximation, Birkhäuser, 1987.

[4] HENRICI P., Applied and Computational Complex Analysis 3, John Wiley \& Sons, New York, 1986.

[5] INOUE T., A remark on zeros of weighted Chebyshev polynomials, Appl. Math. Lett., 4, No.5, (1991), 63-65.

[6] INOUE T., Asymptotic behavior of extremal weighted polynomials, Mathématica, L'acad. Répu. Soc. Rouman, Tome 35(58), No.1 (1993), 29-34.

[7] INOUE T., Equilibrium measure and limit zeros distribution of extremal weighted polynomials, Applied Math. Lett., 7, No. 4 (1994), 75-78. 
[8] INOUE T., Applications of asymptotic theorem on weighted extremal polynomials, Trans. Japan Soc. Ind. App. Math., (in Japanese), 4 (1994), 151-155.

[9] T. INOUE, Fundamental properties of weighted polynomials, Mathématica, L'acad. Répu. Soc. Rouman, Tome 36(59), no. 1 (1994), 33-35.

[10] LANDKOF N.S., Foundation of Modern Potential Theory, Springer, (1972).

[11] MHASKAR H.N., Finite-infinite range inequalities in the complex plane, Internat. J. Math. \& Math. Sci., 14 (1991), 625-638.

[12] MHASKAR H.N. and SAFF E.B., Where does the sup norm of a weighted polynomial live? Constr. Approx., 1 (1985), 71-91.

[13] MHASKAR H.N. and SAFF E.B., Weighted analogues of capacity, transfinite diameter and Chebyshev constant, Constr. Approx., 8 (1992), 105-124.

[14] STAHL H. and TOTIK V., Nth root asymototic behavior of orthogonal polynomials, In: Orthogonal Polynomials, (Nevai P., eds.) Kluwer Acad. Pub., (1990), 395-417.

[15] TREFETHEN L.N.(ed.), Numerical Conformal Mapping, North-Holland, Amsterdam, 1986.

[16] TSUJI M., Potential Theory in Modern Function Theory, 2nd. ed., Chelsea, New York, 1958. 


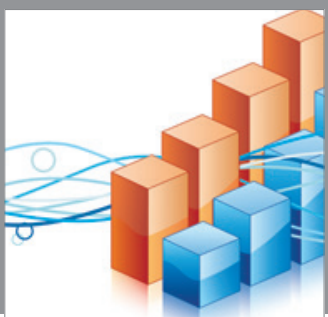

Advances in

Operations Research

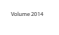

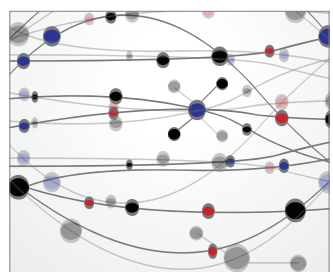

\section{The Scientific} World Journal
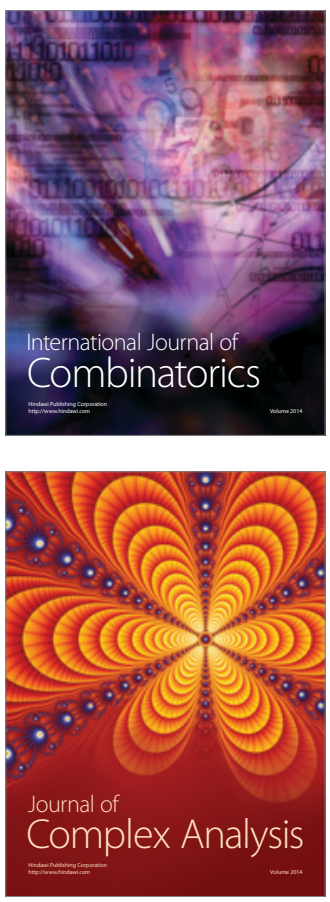

International Journal of

Mathematics and

Mathematical

Sciences
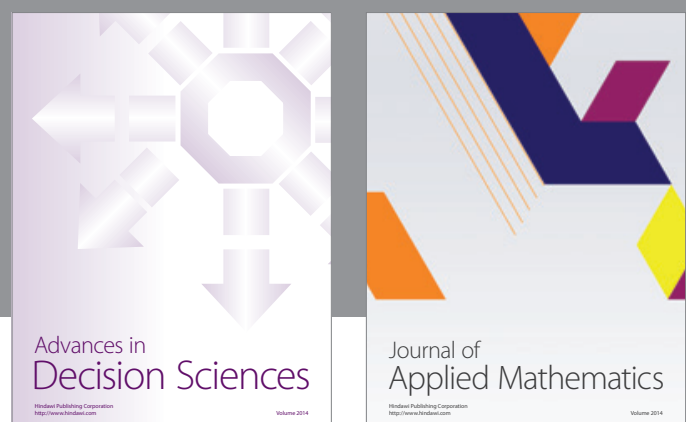

Journal of

Applied Mathematics
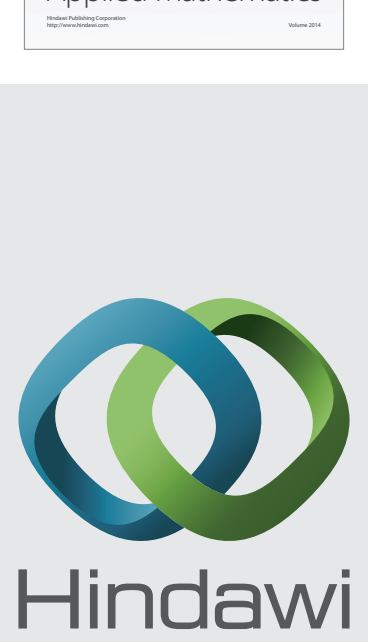

Submit your manuscripts at http://www.hindawi.com
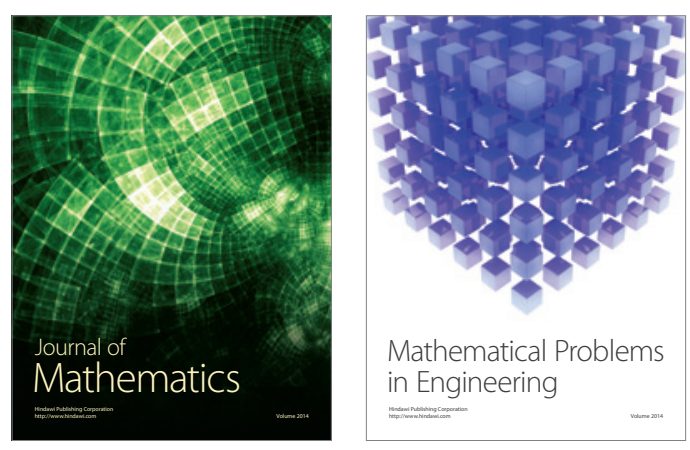

Mathematical Problems in Engineering
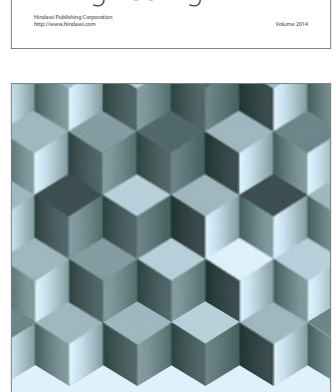

Journal of

Function Spaces
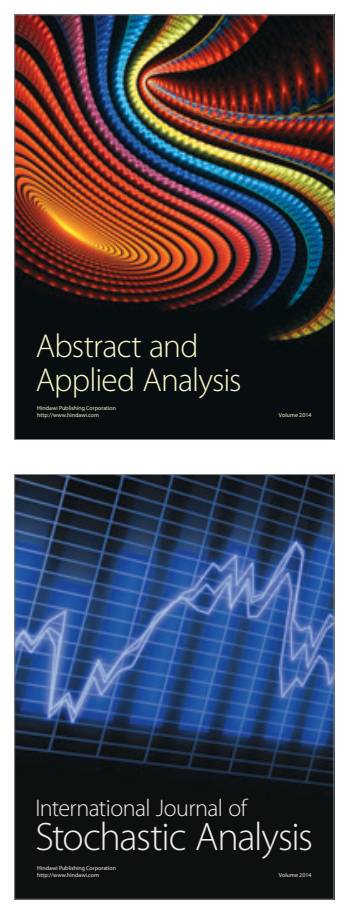

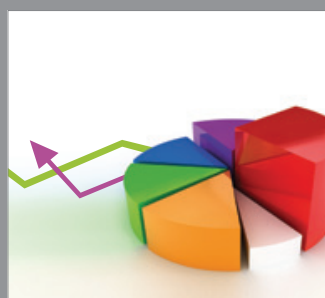

ournal of

Probability and Statistics

Promensencen
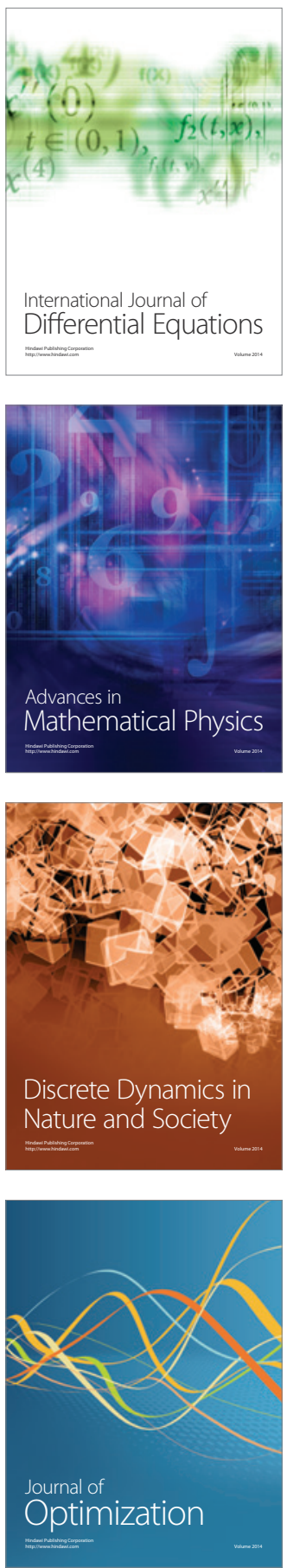